\title{
PHOTOINDUCED CHARGE TRANSFER DYNAMICS AT HYBRID GAAS/P3HT INTERFACES
}

\author{
JUN YIN, MANOJ KUMAR, MAJID PANAHANDEH-FARD AND ZILONG WANG
}

Division of Physics and Applied Physics, School of Physical and Mathematical Sciences, Nanyang Technological University, 21 Nanyang Link, Singapore 637371

\section{FRANCESCO SCOTOGNELLA}

Dipartimento di Fisica, Politecnico di Milano, Piazza Leonardo da Vinci 32, 20133 Milano, Italy

Center for Nano Science and Technology@PoliMi, Istituto Italiano di Tecnologia, Via Giovanni Pascoli 70/3, 20133 Milano, Italy

\section{CESARE SOCI*}

Division of Physics and Applied Physics, School of Physical and Mathematical Sciences, Nanyang Technological University, 21 Nanyang Link, Singapore 637371

Centre for Disruptive Photonic Technologies, Nanyang Technological University, 21 Nanyang Link, Singapore 637371

\section{Introduction}

Organic-inorganic hybrid photovoltaic systems, for instance conjugated polymers blended with inorganic single crystals, thin films, or functionalized nanostructures (nanoparticles, nanorods or quantum dots) [1-2], offer great flexibility for designing novel solar cells with high power conversion efficiency (PCE) [3-5]. The overall photocurrent of hybrid photovoltaics is primarily affected by the competition between interfacial charge separation and charge recombination [6-8]. Typically, the energetic offsets between the frontier orbitals of conjugated polymers and conduction bands (CB) and valence bands (VB) of inorganic nanocrystals make inorganic parts act as electron acceptor,

\footnotetext{
* Corresponding Author: csoci@ntu.edu.sg.
} 
promoting dissociation of the photogenerated excitons and preventing charge recombination [9]. Recently, significant research efforts were directed toward hybrid photovoltaics using different inorganic nanostructure composites of group IV ( $\mathrm{Si}[10]$ and Ge [11]), group IV-VI (PbS [12] and PbSe [13]), group IIVI (CdSe [14-15] and CdS [16]) and metal oxides $\left(\mathrm{TiO}_{2}\right.$ [1] and $\mathrm{ZnO}$ [17-18]). Specifically, mainstream III-V semiconductors (e.g. GaAs and InP) with high carrier mobility and direct bandgap absorption well-overlapped with the solar irradiance are rapidly emerging as exceptional photovoltaic material for thin film technologies [19], including dye or polymer sensitized hybrid solar cells [20-23]. Early demonstration of hybrid photovoltaics based on GaAs/quaterthiophene and GaAs/octithiophene bilayer yielded 1.7\% [20] and $4.2 \%$ PCE [24]; more recently, use of GaAs nanowires either blended in P3HT bulk heterojunctions [21] or in a bilayer thin-film configuration [22, 25] allowed achieving PCEs of $>2.3 \%$.

Despite very promising device performances, current understanding of the electronic properties leading to charge transfer at organic-inorganic interfaces like GaAs/poly(3-hexylthiophene-2,5-diyl) (P3HT) is limited [26-29]. Moreover, the nature of charged photoexcitations at the interface of highly delocalized inorganic crystals and more localized, disordered conjugated systems is of great fundamental interest [30-34]. Based on above perspectives, better understanding of the nature of primary photoexcitations and fundamental physical processes at III-V/polymer heterointerfaces are desirable from both experimental and theoretical standpoints.

Several recent studies of hole and electron transfer mechanisms in IIIV/conjugated polymer systems provided useful insights into interfacial processes of these composites and guidance for the design of efficient photovoltaic devices. Blackburn et al. [15] investigated electron and hole transfer from colloidal InP quantum dots to organic hole-transfer material by photoluminescence and transient absorption spectroscopy. In such system hole transfer induces a rapid $\sim 4$ ps component to the transient absorption (TA) decay, which is manifested in the dynamics of both visible photobleaching and mid-IR absorbance. Lanzani et al. [35] found strong photoinduced absorption signatures in oligothiophene cations upon photoexcitation of a GaAs/oligothiophene bilayer, indicating that charge transfer involves exciton diffusion from oligothiophene bulk to the GaAs/oligothiophene interface. Subsequently, a detailed picture of photoinduced electron transfer at the $\mathrm{GaAs} / \mathrm{CuPcF}_{16}$ interface was also provided [29]. Very recently, Zhu and coworkers revealed the presence of competing charge separation processes at the $\mathrm{GaAs} / \mathrm{CuPc}$ interface by resolving distinct channels of charge carrier separation driven by the 
delocalized space charge field in GaAs and charge transfer from GaAs to localized $\mathrm{CuPc}$ due to an energetic driving force at the interface [8]. In theory, Giustino and coworkers investigated an idealized GaAs(10-10)/P3HT interface by DFT modeling, showing that the resulting interfacial dipole can lower the highest occupied molecular orbital (HOMO) of the conjugated polymer until the whole system attains equilibrium [36]. Our group demonstrated that the polar GaAs(111)B surface tends to facilitate hole transfer from the VB states to the HOMO of P3HT compared to the nonpolar surface GaAs(110) [37].

Here we compare theoretical predictions regarding the charge carrier photogeneration and photoinduced interfacial charge transfer at the heterointerface of a prototype bilayer formed by hole-conducting polymer (P3HT) and $n$-type GaAs substrates with different orientation and polar surfaces by combining steady-state and ultrafast spectroscopy measurements with density functional theory (DFT) calculations. Experimental evidence of efficient electron transfer at $\mathrm{GaAs} / \mathrm{P} 3 \mathrm{HT}$ interfaces is found in photoluminescence (PL) and transient absorption spectra and dynamics with excitation energy above the P3HT optical gap by the enhancement of polaron formation rate and increase of polaron lifetime. On the other hand, selective excitation with energy below the optical gap of P3HT allows isolating the contribution of hole injection from GaAs to P3HT. All these findings are supported by DFT calculations which confirm that, irrespective of the crystal orientation, P3HT acts as an efficient electron donor. On the other hand, the intrinsic surface dipole moment of GaAs surfaces is enhanced by induced charge displacement, facilitating hole transfer to P3HT in the case of GaAs(111)B.

\section{Discussions}

\subsection{Electronic Charge Rearrangement - Density Function Theory Predictions}

The efficiency of electron transfer across GaAs/P3HT interfaces, which ultimately determines charge generation efficiency for photovoltaic applications, is subject to the interfacial energy alignment between the conjugated polymer and the inorganic substrate, the electronic couplings between them, and the change of the polymer geometric and electronic structure due to the thermal fluctuations [38]. Recent theoretical work by Prezhdo et al. described the interfacial charge separation and relaxation processes in hybrid organicinorganic systems by time-domain atomistic simulations [39]. In the case of alizarin $/ \mathrm{TiO}_{2}$ interface, the adiabatic mechanism dominates over non-adiabatic 
ones due to the strong electronic coupling across the interface [40]. For graphene $/ \mathrm{TiO}_{2}$ interface, electron injection is found to be ultrafast due to the strong electronic coupling between graphene and $\mathrm{TiO}_{2}$, and both electron injection and energy transfer accelerate for photoexcited states that are delocalized between the two subsystems [41].

The energy alignment and electronic coupling between a polymer and a semiconductor substrate can be obtained via the analysis of the total density of states (DOS) and of the projected density of states (PDOS) after charge redistribution [42]. For the quantification of the actual charge transfer and separation processes between polymer and semiconductor substrates, the charge redistribution upon P3HT adsorption was evaluated, and the changes of interfacial dipole moment and induced work function estimated [43]. In organic/inorganic heterojunctions, adsorption of organic molecules on inorganic substrates is always accompanied by a certain degree of electronic charge rearrangement across the hybrid interface; as a consequence, the generated dipole moment between the molecule and the inorganic semiconductor caused by charge transfer, offsets the interfacial potential and reduces the energy level mismatch [44-45]. Thus, the nature of the polarity of inorganic surface has substantial effects on the formation of interfacial dipoles and may strongly influence charge separation.

Here the charge transferred from the P3HT molecule to the GaAs substrate was calculated by the difference $\Delta \rho(r)=\rho_{\mathrm{GaAs} / \mathrm{P} 3 \mathrm{HT}}-\left[\rho_{\mathrm{GaAs}}+\rho_{\mathrm{P} 3 \mathrm{HT}}\right]$ ( $r$ is the position vector within the computational cell; $\rho_{\mathrm{GaAs}}$ is the charge density of the $\operatorname{GaAs}(110)$ and (111)B slabs; $\rho_{\mathrm{P} 3 \mathrm{HT}}$ is the charge density of P3HT layer without substrate; and $\rho_{\mathrm{GaAs} / \mathrm{P3HT}}$ is the electronic charge density of the GaAs/P3HT interface). Figures 1(a,a') show that the overall DOS of the GaAs and P3HT hybrid system (dotted line) is largely ruled by the PDOS of GaAs (shaded grey area) while the PDOS of P3HT (solid line) only slightly perturbs the edge of GaAs VB. As compared to the relatively small overlap of the HOMO of P3HT $\left(\mathrm{H}_{\mathrm{P} 3 \mathrm{HT}}\right)$ and the GaAs VB state, the states of P3HT molecule extend over a broad energy range and there is large electronic overlap between the lowest unoccupied molecular orbital (LUMO) level of P3HT ( $\left.\mathrm{L}_{\mathrm{P} 3 \mathrm{HT}}\right)$ and the GaAs CB state, which generates strong electronic coupling. The characterization of orbital overlap across the interface is performed by the analysis of the frontier orbitals of the P3HT molecule interacting with the GaAs substrate. Figures $1\left(b, b^{\prime}\right)$ and 1(c,c') show the spatial electronic distribution at the HOMO and LUMO energies of P3HT. In the case of GaAs(110)/P3HT, electrons may be efficiently transferred to the bulk of the GaAs crystal due to the highly overlapped electron clouds distribution at the interface, indicating the possible formation of hybrid 
delocalized states. On the other hand, hole transfer is unfavorable and in both case would be confined to surface states of GaAs(110) because of the poor overlap between the corresponding $\mathrm{CB}$ and HOMO. In the case of the polar $\operatorname{GaAs}(111) B$ surface, the DOS is severely affected by the presence of surface dangling bonds: As- $4 p$ states of the top surface layer and Ga-4p states of the bottom surface layer lie right at the Fermi level inside the bandgap, leading to a large number of intragap states and significant narrowing of the energy bandgap upon lowering CB energy [46]. Hence the lowering of GaAs(111)B CB has dramatic effects on the relative overlap between GaAs and P3HT states, and changes the resulting electron density distribution. Specifically, the CB of $\mathrm{GaAs}(111) \mathrm{B}$ acquires large overlap with the HOMO of P3HT, which favors the hole transfer from GaAs(111)B to P3HT. While electron transfer from P3HT to GaAs(111)B may be significantly reduced compared to the previous case and somehow confined to the $\mathrm{GaAs}(111) \mathrm{B}$ surface, holes are allowed to delocalize from the thiophene ring over to the GaAs(111)B surface states and deeply into the bulk thanks to the larger coupling.

For both $\mathrm{GaAs}(110)$ and $\mathrm{GaAs}(111) \mathrm{B} / \mathrm{P} 3 \mathrm{HT}$ interfaces, adsorption of P3HT onto the substrate induces significant charge transfer, together with the formation of distinct charge accumulation layers and substantial charge reorganization at the interface. This indicates the significance of electrostatic interaction between the polymer and the substrates [47-48]. The onedimensional plane-averaged charge density difference $(\Delta \rho)$ along $z$-direction shown in Figures $1\left(\mathrm{~d}, \mathrm{~d}^{\prime}\right)$ provides quantitative estimation of electron $(\Delta \rho<0)$ and hole $(\Delta \rho>0)$ accumulation layer, suggesting a much larger charge redistribution in the case of the polar $\operatorname{GaAs}(111) \mathrm{B}$ as compared to the nonpolar $\operatorname{GaAs}(110)$ surface (up to $\sim 5.3 \times 10^{-3} v s \sim 0.8 \times 10^{-3} \mathrm{e} / \AA^{3}$ ). The main difference between such two surfaces is the type of charges accumulated at the interface: holes accumulate both above and below the GaAs surface in nonpolar GaAs(110), while a small electron accumulation layer appears on the top As monolayer in polar GaAs(111)B. This electron accumulation layer reduces electrostatic screening due to the large interfacial holes density and facilitates hole transfer from GaAs to P3HT comparing with the case of nonpolar GaAs(110).

Both bare GaAs(110) and $\operatorname{GaAs}(111) \mathrm{B}$ surfaces have distinct polarization properties; although $\operatorname{GaAs}(110)$ is usually considered as a nonpolar surface, a small intrinsic dipole moment is found due to the tendency of surface $\mathrm{Ga}$ atoms to sink toward the bulk, leaving behind an As-terminated surface. The unbalanced charge at the surface forms a negative dipole moment pointing toward the bulk which, in our simulations, results in a surface charge density of $2.2 \times 10^{13} \mathrm{e} / \mathrm{cm}^{2}$. Unlike the nonpolar $\operatorname{GaAs}(110)$ surface, a large intrinsic dipole 


\section{$\operatorname{GaAs}(110) / \mathbf{P H H T}$}
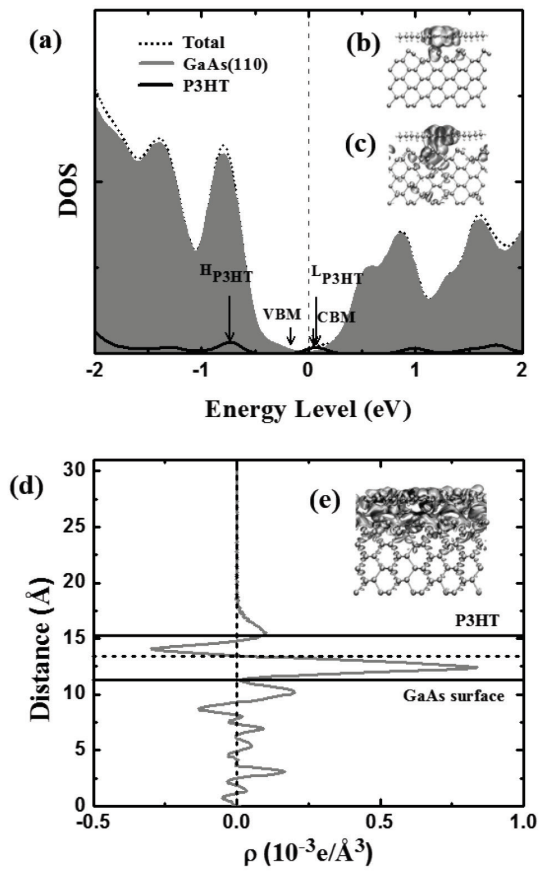

\section{GaAs(111)B/P3HT}
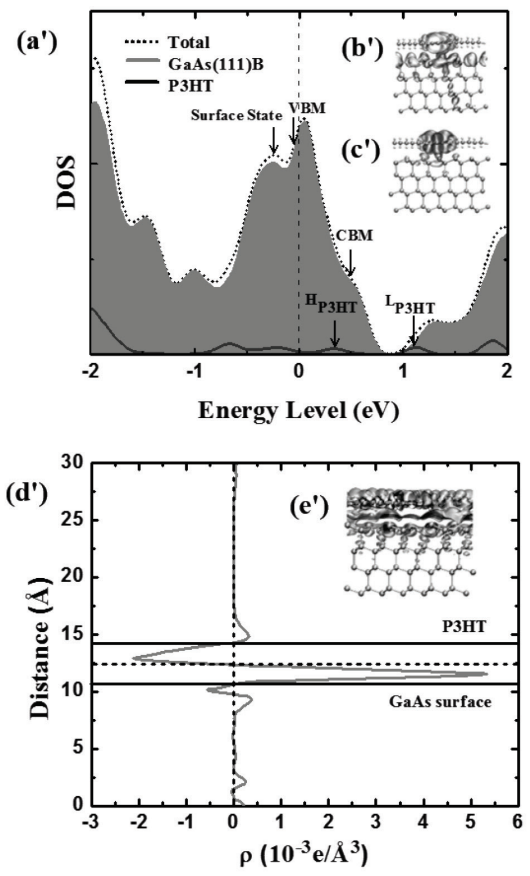

Figure 1. Density of states, electronic orbital distribution and charge redistribution in GaAs(110)/P3HT (left) and GaAs(111)B/P3HT (right) hybrid systems: (a, a') density of states (the dashed line in indicates the position of the Fermi energy); charge distribution of the electron ( $\left.b, b^{\prime}\right)$ and hole (c, c') orbitals; one-dimensional plane-averaged charge density difference upon P3HT adsorption $\left(\mathrm{d}, \mathrm{d}^{\prime}\right)$; three-dimensional representation of the charge density difference with an isovalue of $\pm 0.005 \mathrm{e} / \AA^{3}\left(\mathrm{e}, \mathrm{e}^{\prime}\right)$. The solid lines in $\left(\mathrm{d}, \mathrm{d}^{\prime}\right)$ indicate the average positions of the GaAs surface and the P3HT plane, while the horizontal dashed line shows the interfacial distance at which charge depletion converts into charge accumulation.

moment exists in GaAs(111)B thanks to the alternating As and Ga terminal layers. In this case, we obtained a larger surface charge density of $7.4 \times 10^{13}$ $\mathrm{e} / \mathrm{cm}^{2}$ [49]. Upon charge redistribution with adsorbed P3HT, the intrinsic surface dipole moment of GaAs surfaces is enhanced by the induced charge displacement. To understand the origin of the interfacial dipole moment, a Löwdin charge analysis of the charge density was carried out for these hybrid systems [50]. By comparing the sum of the Löwdin charge on the GaAs and P3HT molecule before and after formation of the interface a total charge $(\Delta Q)$ of $0.207 e$ and $0.209 e$ for $\mathrm{GaAs}(110) / \mathrm{P} 3 \mathrm{HT}$ and $\mathrm{GaAs}(111) \mathrm{B} / \mathrm{P} 3 \mathrm{HT}$ is found to be 
transferred between P3HT and GaAs. These charge values are lower than typical

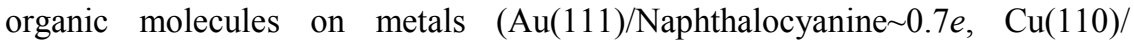
Petencene 0.8e) [51-52], but of the same order of inorganic metaloxide/polymer interfaces $(\mathrm{ZnO} / \mathrm{P} 3 \mathrm{HT} \sim 0.3 e)$ [53], and metal-oxide/graphene interface $(\mathrm{ZnO} / \mathrm{Graphene} 0.4 e)$ [54], which are known to induce significant charge transfer.

\subsection{Energy Alignment-XPS and UPS Measurements}

DFT simulations were validated performing XPS and UPS measurements of the energy alignment of GaAs/P3HT interfaces. According to the integer charge transfer (ICT) model [55], the energy level alignment of organic/inorganic systems with weak interfacial interactions can be determined from the change of work function upon adsorption of the organic molecule. Removing charges from conjugated polymer can induce substantial geometric and electronic relaxation effects, which leads to localized positive polaronic states $(p+)$ [56]. If the work function of substrate $\left(\Phi_{\mathrm{SUB}}\right)$ is larger than the energy of the polaronic states $\left(E_{\mathrm{p}^{+}}\right)$, electrons will spontaneously transfer from the organic layer into the inorganic substrate, creating a dipole that reduces the vacuum level, where the interfacial dipole energy $(\Delta)$ caused by charge redistribution can be obtained from the energy difference between the $\Phi_{\mathrm{SUB}}$ and $E_{\mathrm{p}+}$.

The position of the HOMO level of P3HT is determined to be 1.10 and $0.64 \mathrm{eV}$ below the Fermi energy from the Fermi-edge regions of UPS spectra of P3HT-coated GaAs(110) and GaAs(111)B substrates. Meanwhile, from the cutoff of the UPS spectra, the work function of P3HT on GaAs(100) and $\operatorname{GaAs}(111) \mathrm{B}$ substrates $\left(\Phi_{\mathrm{P} 3 \mathrm{HT} / \mathrm{SUB}}\right)$ are found to be 3.78 and $4.00 \mathrm{eV}$, respectively. To determine the energetics of the bare substrates, the thin P3HT films were removed in-situ by Ar ion sputtering, until exposure of clean GaAs surfaces. GaAs(110) and GaAs(111)B substrates show the valence band maxima (VBM) of 0.69 and $0.68 \mathrm{eV}$ and work functions of 4.76 and $4.95 \mathrm{eV}$. The LUMO level of P3HT is determined by adding the optical gap energy $(1.9 \mathrm{eV})$ to the HOMO; similarly, the CBM of GaAs is the sum of VBM and GaAs optical gap energy (1.42 eV).

The overall picture of energy level alignment determined by the above measurements is sketched in Figures 2(a,a'). A nested configuration (type I) is obtained at the $\operatorname{GaAs}(110) / \mathrm{P} 3 \mathrm{HT}$ interface whereas a staggered band alignment (type II) is observed in the GaAs(111)B/P3HT case, in good agreement with the 
calculated energy alignment in Figure 1(a,a'). Both configurations favor electron transfer from P3HT to GaAs substrates thanks to the barrier between conduction band minimum (CBM) of GaAs and the LUMO of P3HT film. The higher work function of bare GaAs substrates compared to the hybrid GaAs/P3HT systems could be attributed to the built-up of interfacial dipole barrier of -0.98 and -0.95 $\mathrm{eV}$, respectively, which result from the displacement of negative charge from P3HT film to GaAs substrates. On the other hand, only in the case of GaAs(111)B the valence level offset is favorable for the hole injection from the P3HT layer $\left(E_{\mathrm{HOMO}}-E_{\mathrm{VBM}}=0.04 \mathrm{eV}\right)$, suggesting that $\mathrm{P} 3 \mathrm{HT}$ could act as a 'hole acceptor'; opposite behavior is expected for the GaAs(110) surface. The induced vacuum level shift by the interfacial dipole, $\Delta \Phi$, can be calculated from the comparison of the electrostatic potential between the GaAs surface and the P3HT molecular plane using the Helmholtz equation $\Delta \Phi=\mu n / \varepsilon_{0}[43]$ ( $\mu$ is the interface dipole moment, i.e. the amount of excess of charge obtained from Löwdin charge analysis multiplied by the interfacial distance; $n=1 / \mathrm{A}$, while A is the surface area of the interface). Since only one monolayer of P3HT was considered in the simulations, the values of $\Delta \Phi=0.769$ and $0.868 \mathrm{eV}$ obtained for the $\operatorname{GaAs}(111) \mathrm{B}$ and $\mathrm{GaAs}(110)$, respectively, are slightly lower than the interfacial dipole barriers observed experimentally.

(a) $\operatorname{GaAs}(110) / \mathrm{P3HT}$

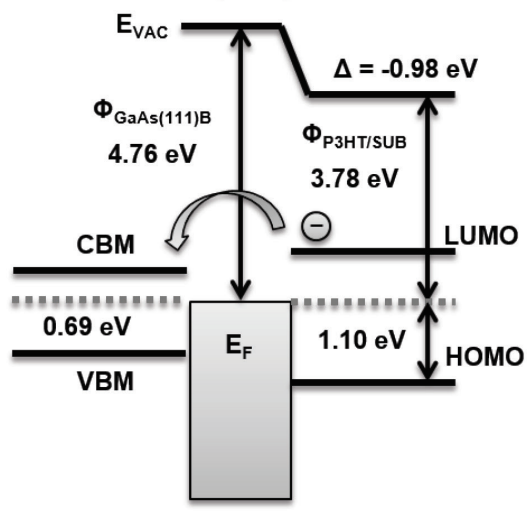

(a') GaAs(111)B/P3HT

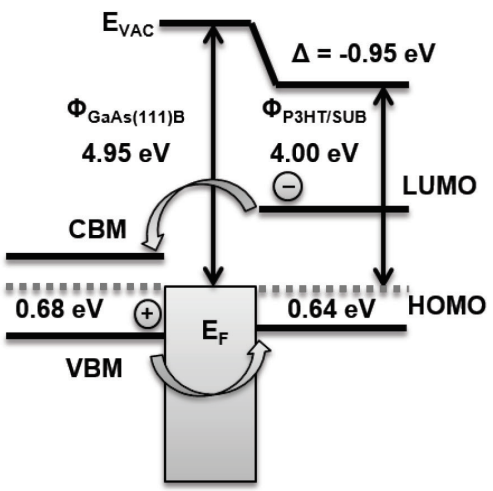

Figure 2. Schematic of the energy diagrams determined from UPS measurements of $\operatorname{GaAs}(110) / \mathrm{P} 3 H T$ (a) and GaAs(111)B/P3HT (a') heterointerfaces. 


\subsection{Charge Transfer Efficiency - Steady-State Spectroscopy Measurements}

The effects of charge transfer across the hybrid interface are illustrated by the optical absorption and photoluminescence spectra of the combined systems. In the top panel of Figure 3(a) the reflectance of $\mathrm{GaAs}(111) \mathrm{B}$ substrate is superimposed to the absorption of P3HT film: the reflectance spectrum of $\mathrm{GaAs} / \mathrm{P} 3 \mathrm{HT}$ is nearly flat in the entire visible region and the typical vibronic features of pristine P3HT film [57] can be clearly resolved from the reflectance spectrum. Quenching of the PL intensity at steady-state provides a clear evidence of interfacial charge transfer. As shown in the bottom panel of Figure 3(a), the PL spectrum of the GaAs/P3HT shows main peaks originating from P3HT with vibronic features at 660 and $720 \mathrm{~nm}$ [58] and from GaAs substrate with emission peak at $875 \mathrm{~nm}$. However, the PL intensity of P3HT in $\mathrm{GaAs} / \mathrm{P} 3 \mathrm{HT}$ is reduced compared to pristine P3HT with the same film thicknesses. Quenching of the P3HT PL suggests the reduction of exciton population upon charge transfer [58-59]. Both emission and absorption spectra of GaAs/P3HT bilayer suggest that GaAs acts both as effective electron acceptor and light absorber in the combined system.

(a)

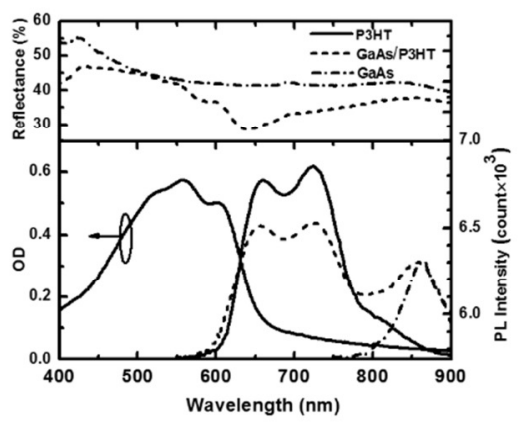

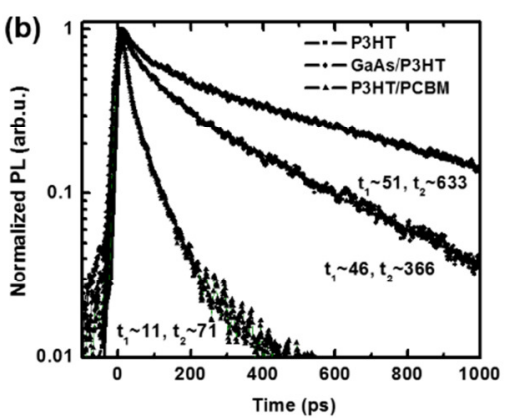

Figure 3. (a) Top panel: reflectance of GaAs (dash-dotted line) and GaAs/P3HT (dashed line); Bottom panel: absorption and steady-state photoluminescence spectra of P3HT (solid lines), and photoluminescence spectra of GaAs/P3HT bilayer (dashed line), and GaAs substrate (dash-dotted line). (b) Time resolved PL of pristine P3HT film (squares), P3HT/PCBM blend film (triangles), and GaAs/P3HT bilayer (circles). The time resolved PL spectra were detected at $660 \mathrm{~nm}$ with photoexcitation at $500 \mathrm{~nm}$. The PL decay dynamics were fitted to a bioexponential function; $\mathrm{t}_{1}$ and $\mathrm{t}_{2}$ are the extracted lifetimes. Thickness of the P3HT films used in both absorption and PL measurements was $40 \mathrm{~nm}$. 
The observed reduction of PL lifetime of P3HT is due to effective exciton dissociation at the GaAs/P3HT interface and photoinduced charge transfer between P3HT and GaAs. The charge transfer efficiency can be estimated using the exciton lifetimes extracted from Figure 3(b). The relative charge transfer efficiency of the combined system can be estimated from the ratio of the fast components of the radiative decay to that of P3HT/PCBM [58]. The estimated charge transfer rate of GaAs/P3HT is found to be $\sim 3 \%$ of that of $\mathrm{P} 3 \mathrm{HT} / \mathrm{PCBM}$ blend film, which is known to have efficiency of $\sim 100 \%$ [60]. This shows that the GaAs/P3HT system also facilitates dissociation of photogenerated excitons and subsequently interfacial transfer by the formation of hybrid interfacial states.

\subsection{Photogenerated Carrier Dynamics - Ultrafast Spectroscopy Measurements}

Femtosecond pump-probe transient absorption measurements were performed in reflection geometry in the range of $500-900 \mathrm{~nm}$ to investigate the prompt dynamics of photogenerated species with different excitation energies. Here the negative features in differential transient reflectance (TR) spectra (note $-\Delta R / R$ on the vertical axis) correspond to photobleaching (PB) or stimulated emission (SE) from excited states and positive features correspond to photoinduced absorption (PIA) mainly due to polarons [61].

Transient absorption dynamics at three significant probe wavelengths (namely 500, 650 and $900 \mathrm{~nm}$ ) are reported in Figures 4 and 5. The dynamics in Figure 4 were obtained using the second harmonic of a Ti:Sapphire amplified system $\left(\lambda_{\mathrm{ex}}=400 \mathrm{~nm}\right)$, while those in Figure 5 using the fundamental of the Ti:Sapphire $\left(\lambda_{\mathrm{ex}}=800 \mathrm{~nm}\right)$ as excitation. Samples were excited with energy per pulse of $0.3 \mu \mathrm{J}$. Both Figures 4 and 5 show a comparison between the dynamics of $\mathrm{GaAs}(110) / \mathrm{P} 3 \mathrm{HT}$ (left) and $\mathrm{GaAs}(111) \mathrm{B} / \mathrm{P} 3 \mathrm{HT}$ (right) hybrid systems to highlight the differences arising from the two crystallographic orientations (surface polarity) of GaAs.

Dynamic traces obtained with excitation wavelength at $400 \mathrm{~nm}$ in the different probe spectral regions (Figure 4) are attributed based on transient reflectance (TR) spectra (not shown here). GaAs(110) and GaAs(111)B/P3HT systems show similar dynamic features: $\mathrm{PB}$ and PIA signals are long-lived $(>500 \mathrm{ps}$ ), consistent with the formation of charged polaron states in P3HT. The rise time of PIA correlates well with the fast decay time of PB signals due to the depletion of the ground state upon charge transfer between P3HT and GaAs and dissociation of singlet excitons. The prolonged PB signal also indicates small 
repopulation of excited states following charge transfer. The main difference between the dynamics of the two hybrid systems appears in the characteristic PB signal at $500 \mathrm{~nm}$. In the case of GaAs(111)B/P3HT the PB signal at $500 \mathrm{~nm}$ decays quickly within the first $200 \mathrm{fs}$ and then remains almost unchanged up to the ns time scale. The initial decay correlates well with the rise of the long-lived PIA signal at $650 \mathrm{~nm}$ due to the formation of polarons in P3HT. In the case of GaAs(110)/P3HT, the signal at $500 \mathrm{~nm}$ nearly instantaneously decays to $\sim 17 \%$ of its initial amplitude and becomes positive after approximately 1 ps due to the predominant contribution from GaAs. These observations correlate well with our theoretical prediction that electron transfer from $\mathrm{P} 3 \mathrm{HT}$ to GaAs is expected in both hybrid systems, but largely favored in the case of GaAs(111)B polar surface.

Ultrafast spectroscopy data and energy considerations suggest that prompt formation of charges at the GaAs/P3HT heterointerfaces may be induced by either electron transfer from $\mathrm{P} 3 \mathrm{HT}$ to GaAs or by hole injection from $\mathrm{GaAs}$ to P3HT. To address this issue, the excitation energy was reduced to below the optical gap of P3HT. In this case, selectively excitation of GaAs allows isolating the contribution of hole injection from GaAs to P3HT. Figure 5 shows the transient dynamics obtained with excitation wavelength of $800 \mathrm{~nm}$. One- and two-photon absorption in P3HT was ruled out at these optical fluences from the absence of any photoluminescence or SE from the polymer. As a result very weak signals are observed at 650 and $900 \mathrm{~nm}$, while the PB previously seen at $500 \mathrm{~nm}$ is absent. The TR spectra of GaAs/P3HT bilayers excited at $800 \mathrm{~nm}$ show similar features as for photoexcitation at $400 \mathrm{~nm}$ (not shown here). Notably, even without direct excitation of the polymer, PIA features of P3HT are clearly visible. In the case of $\mathrm{GaAs}(111) \mathrm{B}$, the long-lived (up to ns) charged polarons corresponding to the PIA signals probed at 650 strongly indicate interfacial charge generation upon hole injection from GaAs(111)B to P3HT, with a final state that coincides with the electron transfer state from P3HT to $\mathrm{GaAs}(111) \mathrm{B}$. Therefore, upon selective excitation of $\mathrm{GaAs}(111) \mathrm{B}$, holes are efficiently injected into P3HT within a time span of $t<1$ ps. Conversely, GaAs(110)/P3HT shows smaller PIA at $650 \mathrm{~nm}$, with somehow faster decays. This indicates that hole transfer is unfavorable compared to the case of the polar $\mathrm{GaAs}(111) \mathrm{B}$ orientation, consistent with the theoretical predictions and UPS measurements discussed earlier. 
$\operatorname{GaAs}(110) / P 3 H T$
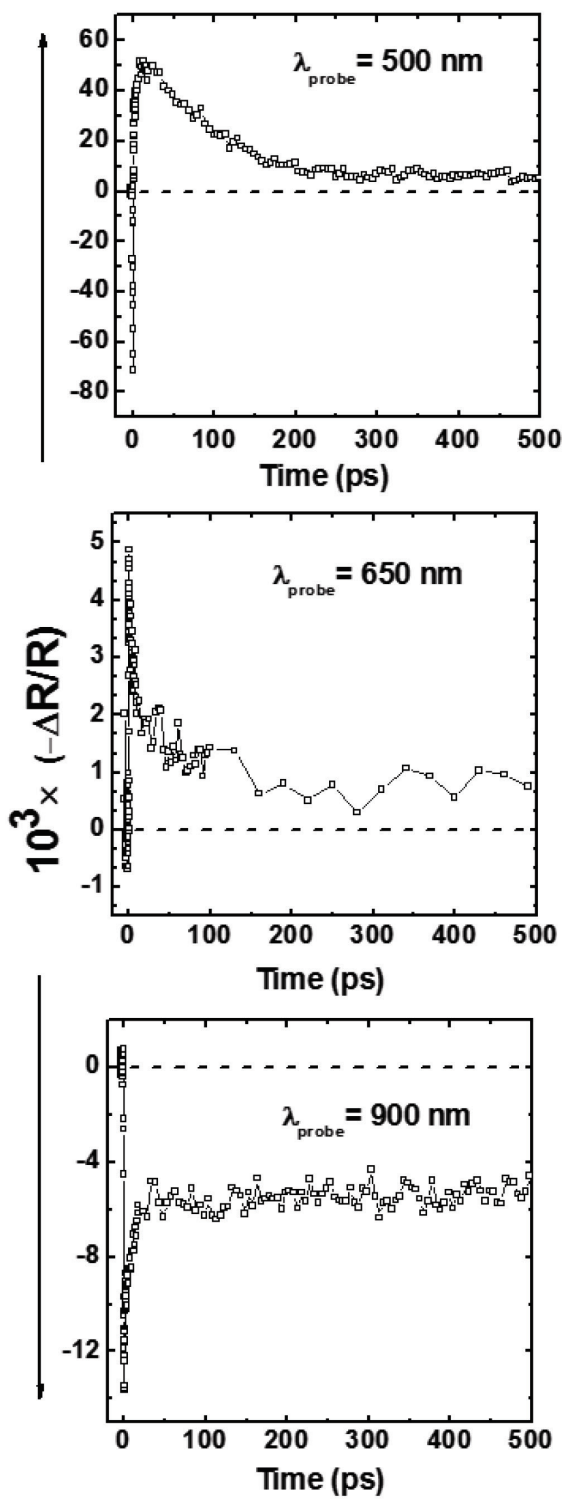

GaAs(111)B/P3HT
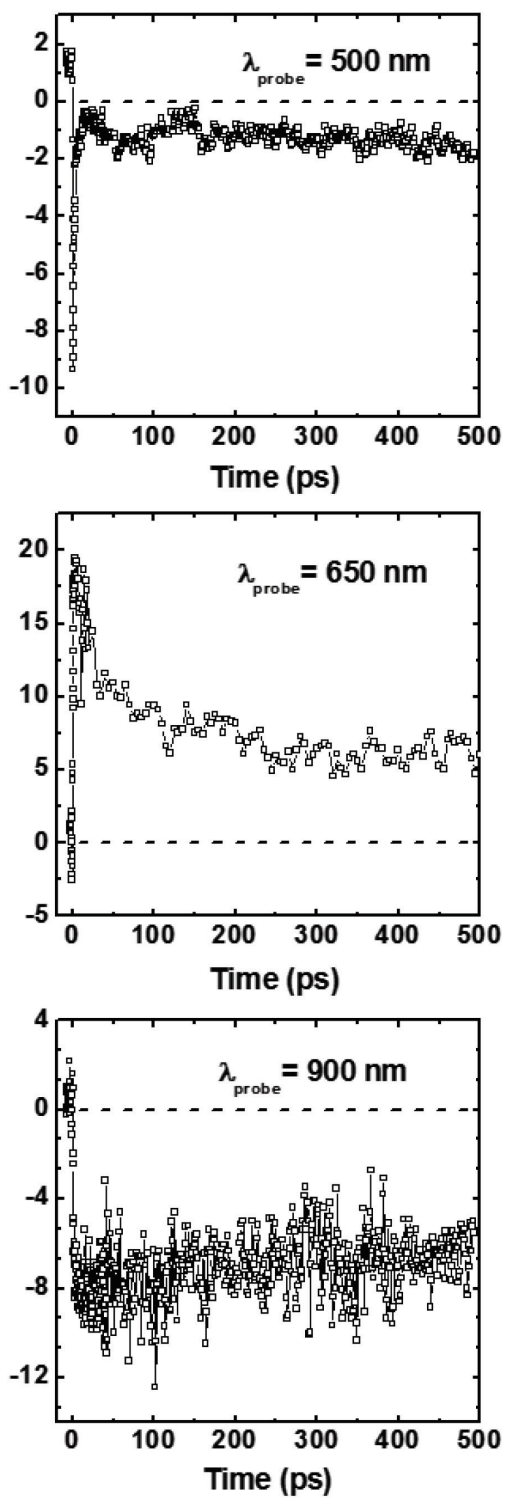

Figure 4. Transient reflection decay profiles of GaAs(110)/P3HT (left) and GaAs(111)B/P3HT (right), at variuos probe wavelengths: photobleaching (PB) at $500 \mathrm{~nm}$, photoinduced absorption (PIA) at $650 \mathrm{~nm}$, and $\mathrm{PB}$ at $900 \mathrm{~nm}$. Excitation wavelength: $400 \mathrm{~nm}$. 
GaAs(110)/P3HT
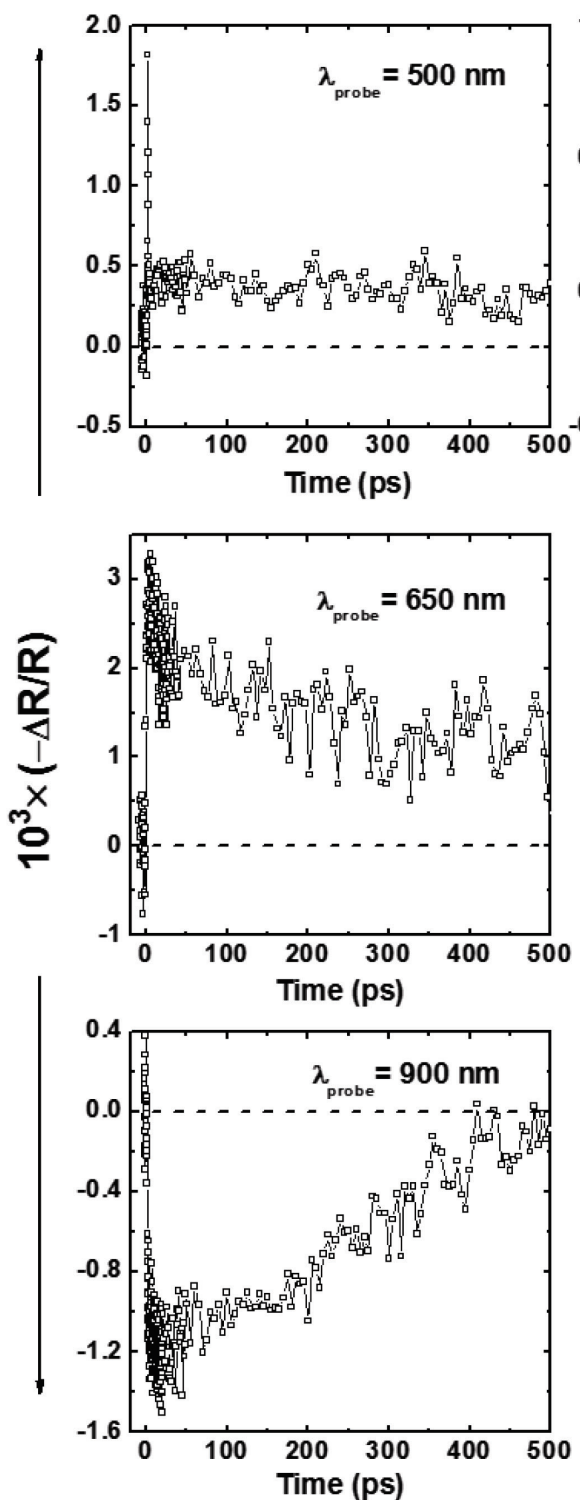

GaAs(111)B/P3HT
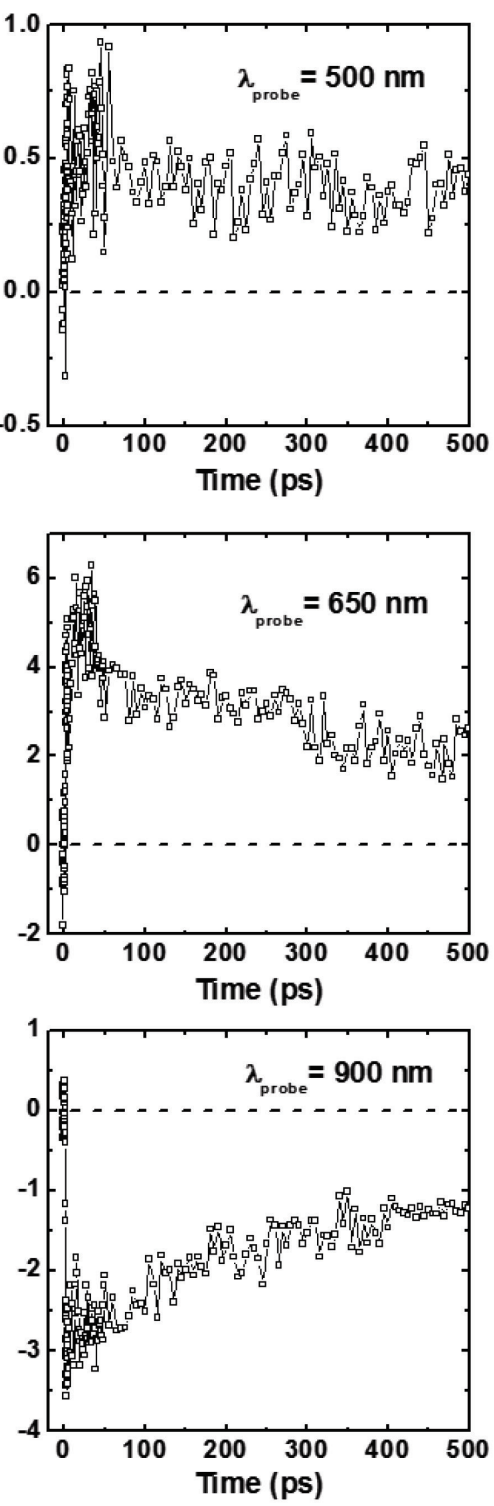

Figure 5. Transient reflection decay profiles of GaAs(110)/P3HT (left) and GaAs(111)B/P3HT (right), at variuos probe wavelengths: photobleaching $(\mathrm{PB})$ at $500 \mathrm{~nm}$, photoinduced absorption (PIA) at $650 \mathrm{~nm}$, and $\mathrm{PB}$ at $900 \mathrm{~nm}$. Excitation wavelength: $800 \mathrm{~nm}$. 


\section{Conclusions}

We investigated charge carrier photogeneration and transfer in hybrid GaAs/P3HT bilayers with different surface orientation (polarity) by using DFT calculations, photoemission spectroscopy, and ultrafast spectroscopy measurements. The combination of experimental data and theoretical predictions provides clear evidence for interfacial charge transfer of both electrons and holes at the heterointerface. DFT calculations show that the different polarity of the GaAs surface affects the electronic orbital and charge redistribution properties. Both $\mathrm{GaAs}(110) / \mathrm{P} 3 \mathrm{HT}$ and $\mathrm{GaAs}(111) \mathrm{B} / \mathrm{P} 3 \mathrm{HT}$ favor electron transfer from P3HT to GaAs substrates. Substantial surface dipole moments $(\Delta \mathrm{Q}$ $\approx 0.21 \mathrm{e}$ ) may be induced on the GaAs surfaces, which promotes charge transfer across the interface. The experimental transient absorption measurements of GaAs/P3HT bilayers show clear signatures of both electron and hole transfer through the presence of large populations of long-lived polarons in PIA signal. Selective excitation of GaAs in GaAs(111)B/P3HT bilayers shows that hole injection from GaAs(111)B to P3HT induces long-lived ( ns) PB and PIA, which is reduced in the case of GaAs(110).

Our theoretical predictions are in agreement with the experimental findings, and provide a robust framework to understand and design hybrid photovoltaic systems. To increase charge separation and reduce charge recombination, large interfacial areas in contact with the organic materials will be required when designing hybrid solar cells. Low-dimensional compounds, such as GaAs nanowires or quantum dots may then be effectively incorporated in hybrid organic-inorganic devices, providing a large extent of flexibility for surface, bandgap, and DOS engineering.

\section{Acknowledgments}

We are grateful to Dr. Shi Chen and Prof. Alfred Huan for their help with photoelectron spectroscopy, and to Jefri Teguh for time-resolved photoluminescence measurements. Thanks also to Prof. Guglielmo Lanzani and Dr. Dmitri Migas for the fruitful discussions regarding this work. C.S. acknowledges financial support from the Nanyang Assistant Professorship, F.S. from the Tan Chin Tuan Fellowship, and M.P.F. from the Yousef Jameel Scholarship. 


\section{References}

1. W. U. Huynh; J. J. Dittmer; A. P. Alivisatos, Science 295, 2425 (2002).

2. Y. Yin; A. P. Alivisatos, Nature 437, 664 (2005).

3. C. Y. Liu; Z. C. Holman; U. R. Kortshagen, Nano Lett. 9, 449 (2009).

4. J. A. Chang; J. H. Rhee; S. H. Im; Y. H. Lee; H. J. Kim; S. I. Seok; M. K. Nazeeruddin; M. Gratzel, Nano Lett. 10, 2609 (2010).

5. S. Dayal; N. Kopidakis; D. C. Olson; D. S. Ginley; G. Rumbles, Nano Lett. 10, 239 (2010).

6. Y. Vaynzof; D. Kabra; L. H. Zhao; P. K. H. Ho; A. T. S. Wee; R. H. Friend, Appl. Phys. Lett. 97, 033309 (2010).

7. Chemsuschem 3,1103 (2010).

8. H. Park; M. Gutierrez; X. Wu; W. Kim; X. Y. Zhu, J. Phys. Chem. C 117, 10974 (2013).

9. P. Vanlaeke; A. Swinnen; I. Haeldermans; G. Vanhoyland; T. Aernouts; D. Cheyns; C. Deibel; J. D'Haen; P. Heremans; J. Poortmans et al., Sol. Energy Mater. Sol. Cells 90, 2150 (2006).

10. L. N. He; Rusli; C. Y. Jiang; H. Wang; D. Lai, IEEE Electron Device Lett. 32, 1406 (2011).

11. A. Du Pasquier; D. D. T. Mastrogiovanni; L. A. Klein; T. Wang; E. Garfunkel, Appl. Phys. Lett. 91, 183501 (2007).

12. S. Gunes; K. P. Fritz; H. Neugebauer; N. S. Sariciftci; S. Kumar; G. D. Scholes, Sol. Energy Mater. Sol. Cells 91, 420 (2007).

13. D. Cui; J. Xu; T. Zhu; G. Paradee; S. Ashok; M. Gerhold, Appl. Phys. Lett. 88, 183111 (2006).

14. Y. Zhou; F. S. Riehle; Y. Yuan; H.-F. Schleiermacher; M. Niggemann; G. A. Urban; M. Kruger, Appl. Phys. Lett. 96, 013304 (2010).

15. J. L. Blackburn; D. C. Selmarten; R. J. Ellingson; M. Jones; O. Micic; A. J. Nozik, J. Phys. Chem. B 109, 2625 (2005).

16. S. Ren; L.-Y. Chang; S.-K. Lim; J. Zhao; M. Smith; N. Zhao; V. Bulovic; M. Bawendi; S. Gradecak, Nano Lett. 11, 3998 (2011).

17. W. J. E. Beek; M. M. Wienk; R. A. J. Janssen, Adv. Funct. Mater. 16, 1112 (2006).

18. S. D. Oosterhout; M. M. Wienk; S. S. van Bavel; R. Thiedmann; L. J. A. Koster; J. Gilot; J. Loos; V. Schmidt; R. A. J. Janssen, Nat. Mater. 8, 818 (2009).

19. E. Yablonovitch; O. D. Miller; S. R. Kurtz; Ieee. The Opto-Electronic Physics That Broke the Efficiency Limit in Solar Cells. Ieee: New York, 2012; p 1556.

20. J. Ackermann; C. Videlot; A. El Kassmi, Thin Solid Films 403, 157 (2002).

21. S. Q. Ren; N. Zhao; S. C. Crawford; M. Tambe; V. Bulovic; S. Gradecak, Nano Lett. 11, 408 (2011).

22. J. J. Chao; S. C. Shiu; C. F. Lin, Sol. Energy Mater. Sol. Cells 105, 40 (2012). 
23. G. Mariani; R. B. Laghumavarapu; B. T. de Villers; J. Shapiro; P. Senanayake; A. Lin; B. J. Schwartz; D. L. Huffaker, Appl. Phys. Lett. 97, 013107 (2010).

24. J. Ackermann; C. Videlot; A. El Kassmi; R. Guglielmetti; F. Fages, Adv. Funct. Mater. 15, 810 (2005).

25. L. Yan; W. You, Acs Nano 7, 6619 (2013).

26. C. K. Yong; K. Noori; M. Gao; H. J. Joyce; H. H. Tan; C. Jagadish; F. Giustino; M. B. Johnston; L. M. Herz, Nano Lett. 12, 6293 (2012).

27. H. Park; M. Gutierrez; X. Wu; J. W. Kim; X. Zhu, J. Phys. Chem.C, 117, 10974 (2013).

28. J. Cabanillas-Gonzalez; A. Gambetta; M. Zavelani-Rossi; G. Lanzani, Appl. Phys. Lett. 91, 122113 (2007).

29. J. Cabanillas-Gonzalez; H.-J. Egelhaaf; A. Brambilla; P. Sessi; L. Duò; M. Finazzi; F. Ciccacci; G. Lanzani, Nanotechnology 19, 424010 (2008).

30. F. Bassani; G. C. La Rocca; D. M. Basko; V. M. Agranovich, Phys. Solid State 41, 701 (1999).

31. S. Blumstengel; S. Sadofev; C. Xu; J. Puls; F. Henneberger, Phys. Rev. Lett. 97, 237401 (2006).

32. Q. Zhang; T. Atay; J. R. Tischler; M. S. Bradley; V. Bulovic; A. V. Nurmikko, Nat. Nanotechnol. 2, 555 (2007).

33. S. Nizamoglu; X. W. Sun; H. V. Demir, Appl. Phys. Lett. 97, 263106 (2010).

34. C. K. Yong; H. J. Joyce; J. Lloyd-Hughes; Q. Gao; H. H. Tan; C. Jagadish; M. B. Johnston; L. M. Herz, Small 8, 1725 (2012).

35. J. Cabanillas-Gonzalez; A. Gambetta; M. Zavelani-Rossi; G. Lanzani, Appl. Phys. Lett. 91, (2007).

36. C. K. Yong; K. Noori; Q. Gao; H. J. Joyce; H. H. Tan; C. Jagadish; F. Giustino; M. B. Johnston; L. M. Herz, Nano Lett. 12, 6293 (2012).

37. J. M. Yin, D. B.; Panahandeh-Fard, M.; Chen, S.; Wang ZL.; Lova P.; and Soci, C. , J. Phys. Chem. Lett. 4, 3303 (2013).

38. S. Dag; L. W. Wang, Nano. Lett. 8, 4185 (2008).

39. A. V. Akimov; A. J. Neukirch; O. V. Prezhdo, Chem. Rev. 113, 4496 (2013).

40. W. R. Duncan; C. F. Craig; O. V. Prezhdo, J. Am. Chem. Soc. 129, 8528 (2007).

41. R. Long; N. J. English; O. V. Prezhdo, J. Am. Chem. Soc. 134, 14238 (2012).

42. S. Nenon; R. Mereau; S. Salman; F. Castet, J. Phys. Chem. Lett. 3, 58 (2012).

43. N. Sai; R. Gearba; A. Dolocan; J. R. Tritsch; W. L. Chan; J. R. Chelikowsky; K. Leung; X. Y. Zhu, J. Phys. Chem. Lett. 3, 2173 (2012).

44. H. Ishii; K. Sugiyama; E. Ito; K. Seki, Adv. Mater. 11, 972 (1999). 
45. X. Crispin; V. Geskin; A. Crispin; J. Cornil; R. Lazzaroni; W. R. Salaneck; J. L. Bredas, J. Am. Chem. Soc. 124, 8131 (2002).

46. L. Lin; J. Robertson, Appl. Phys. Lett. 98, 082903 (2011).

47. H. Nemec; J. Rochford; O. Taratula; E. Galoppini; P. Kuzel; T. Polivka; A. Yartsev; V. Sundstrom, Phys. Rev. Lett. 104, 197401 (2010).

48. O. L. A. Monti, J. Phys. Chem. Lett. 3, 2342 (2012).

49. F. Bernardini; V. Fiorentini; D. Vanderbilt, Phys. Rev. B 56, 10024 (1997).

50. P. Giannozzi; S. Baroni; N. Bonini; M. Calandra; R. Car; C. Cavazzoni; D. Ceresoli; G. L. Chiarotti; M. Cococcioni; I. Dabo et al., J. Phys.: Condens. Matter 21, 395502 (2009).

51. A. Terentjevs; M. P. Steele; M. L. Blumenfeld; N. Ilyas; L. L. Kelly; E. Fabiano; O. L. A. Monti; F. Della Sala, J. Phys. Chem. C 115, 21128 (2011).

52. K. Muller; A. P. Seitsonen; T. Brugger; J. Westover; T. Greber; T. Jung; A. Kara, J. Phys. Chem. C 116, 23465 (2012).

53. K. Noori; F. Giustino, Adv. Funct. Mater. 22, 5089 (2012).

54. Z. Geng. W., X. F. , Liu. H. X. , Yao. X. J., J. Phys. Chem. C 117, 10536 (2013).

55. S. Braun; W. R. Salaneck; M. Fahlman, Adv. Mater. 21, 1450 (2009).

56. Z. Xu; L. M. Chen; M. H. Chen; G. Li; Y. Yang, Appl. Phys. Lett. 95, 013301 (2009).

57. P. Salek; O. Vahtras; J. D. Guo; Y. Luo; T. Helgaker; H. Agren, Chem. Phys. Lett. 374, 446 (2003).

58. D. Jarzab; K. Szendrei; M. Yarema; S. Pichler; W. Heiss; M. A. Loi, $A d v$. Funct. Mater. 21, 1988 (2011).

59. J. Piris; T. E. Dykstra; A. A. Bakulin; P. H. M. van Loosdrecht; W. Knulst; M. T. Trinh; J. M. Schins; L. D. A. Siebbeles, J. Phys. Chem. C 113, 14500 (2009).

60. J. M. Guo; H. Ohkita; H. Benten; S. Ito, J. Am. Chem. Soc. 132, 6154 (2010).

61. F. Deschler; A. De Sio; E. von Hauff; P. Kutka; T. Sauermann; H. J. Egelhaaf; J. Hauch; E. Da Como, Adv. Funct. Mater. 22, 1461 (2012). 